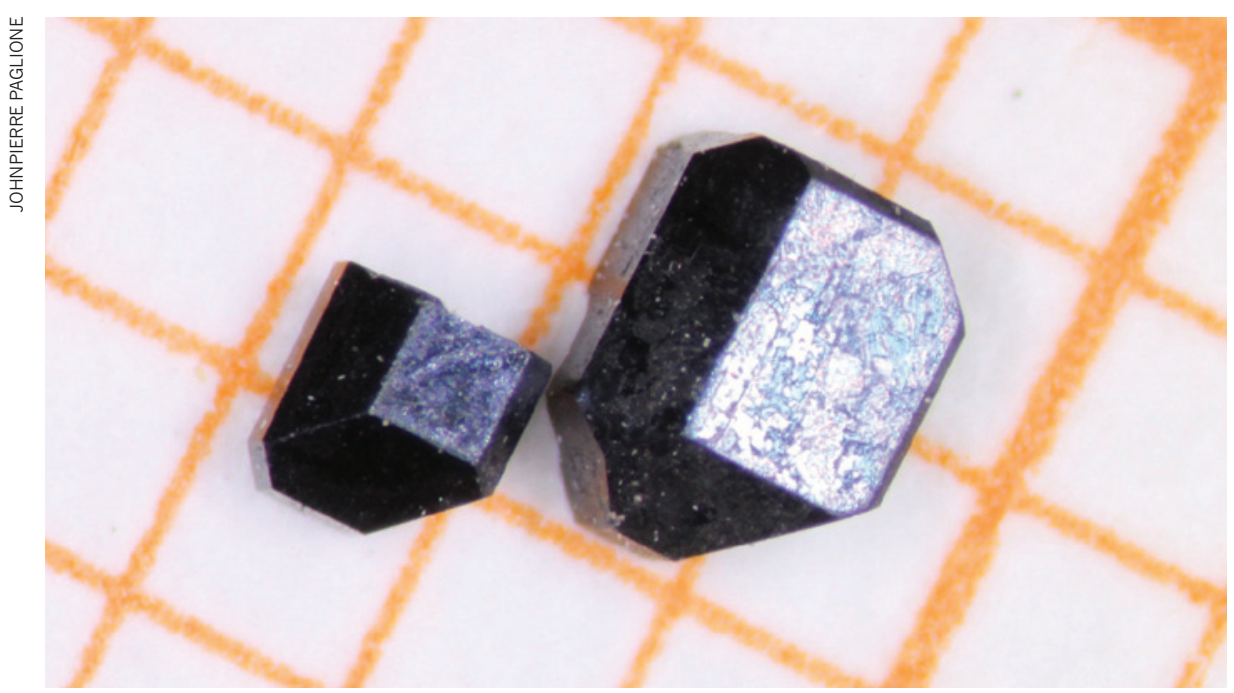

Despite being insulators inside, samarium hexaboride crystals can conduct electricity on their surface.

CONDENSED-MATTER PHYSICS

\title{
Hopes surface for exotic insulator
}

\section{Findings by three teams may solve a 40-year-old mystery.}

\section{BY EUGENIE SAMUEL REICH}

A compound whose odd electrical behaviour has puzzled physicists for decades could turn out to be a boon for quantum physics and electronic-device makers.

When theorists proposed in 2005 that it should be possible to find materials that conduct electricity at the surface while the rest of the sample behaves as an insulator, physicists were intrigued. They wanted to study the quantum effects that should emerge in such materials, and to explore applications in low-power electronics and quantum computing. But topological insulators, as the materials were called, proved fiendishly difficult to make. Some researchers have slaved to produce thin films using complex techniques that are unlikely ever to scale up to the levels needed for industrial purposes. Others have contented themselves with compounds that approximate topological insulators but still have a degree of internal conductivity.

Now, three papers ${ }^{1-3}$ suggest that samarium hexaboride, a poorly understood compound that was first found to gain conducting properties at very low temperatures ${ }^{4}$ in 1969 by researchers at Bell Labs in New Jersey, may in fact be a topological insulator in its bulk form.

In the most recent paper ${ }^{1}$ in the trend, posted online on 28 November, researchers at the University of California, Irvine, report seeing remarkably fast-moving electrons on the surface of $\mathrm{SmB}_{6}$ crystals, which they take as a sign of a superb surface conductor. Five days earlier, researchers at the University of Maryland in College Park had reported measurements tracing the path of electrons injected into $\mathrm{SmB}_{6}$ samples as they were cooled ${ }^{2}$. Those results suggest that the material is insulating in its interior at temperatures below around 30 kelvin. And, in a paper posted on 21 November $^{3}$, scientists from the University of Michigan in Ann Arbor and University of California, Irvine, describe their measurements of conductivity through the surface and bulk of the material, and find evidence that the

\section{CHARGING UP}

The number of papers published on topological insulators has grown rapidly over the past few years

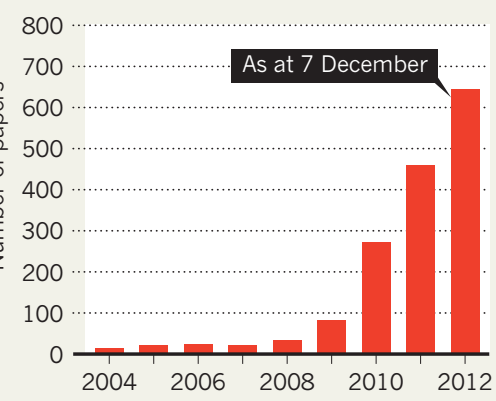

surface conducting behaviour persists despite imperfections and impurities, as would be expected from a true topological insulator.

A spurt of interest in topological insulators over the past few years (see 'Charging up') led to a 2010 prediction that $\mathrm{SmB}_{6}$ would be such a material ${ }^{5}$. "I'd say we've been tentatively vindicated," says Piers Coleman of Rutgers University in Piscataway, New Jersey, one of the four theoretical physicists who made the prediction. "We're thrilled by these new results."

\section{COOL CHARACTERISTICS}

The prediction grew, in part, from studies of materials known as Kondo insulators, which, unlike ordinary insulators, retain some of the small amount of conductivity they do have when they are cooled to a few degrees above absolute zero. $\mathrm{SmB}_{6}$, which is often categorized as a Kondo insulator, fits this description.

Coleman and other theorists realized that the material's behaviour would make sense if it were a topological insulator. That would mean that the quantum properties of the material would be such that electrons cannot flow through it freely, as they would in an ordinary conductor, except at the material's surface. If this proves correct, Coleman thinks that insights gleaned from $\mathrm{SmB}_{6}$ and other Kondo insulators could carry over to all topological insulators.

$\mathrm{SmB}_{6}$ is an unusual topological insulator because the electrons in the outer shells of the samarium atoms interact with one another strongly, such that a coordinated motion emerges. This could make the material useful for creating some exotic quantum effects, including magnetic monopoles, or Majorana fermions - quasiparticles that might be useful for quantum computing, says Shoucheng Zhang, who has pioneered work on topological insulators at Stanford University in California. Zhang adds that the rush of interest in $\mathrm{SmB}_{6}$ is part of a trend to study materials with electrons that interact strongly with each other. "Now we're looking at a number of systems. It's a very exciting development," he says.

Peter Armitage, who has been working on topological insulating behaviour in bismuthbased compounds at John Hopkins University in Baltimore, Maryland, says that in the field of condensed-matter physics, experiment usually leads theory, but this is a remarkable example of the opposite. He is now hoping to start experiments on $\mathrm{SmB}_{6}$ in the next week or two to confirm and study the surface states. "These are beautiful effects that were hiding under our noses," he says. "This is a very big advance." -

1. Botimer, J. et al. Preprint at http://arxiv.org/ abs/1211.6769 (2012)

2. Zhang, X. et al. Preprint at http://arxiv.org/ abs/1211.5532 (2012).

3. Wolgast, S. 5 al. Preprint at http://arxiv.org/ abs/1211.5104 (2012)

4. Menth, A., Buehler, E. \& Geballe, T. H. Phys. Rev. Lett 22, 295-297 (1969)

5. Dzero, M., Sun, K., Galitski, V. \& Coleman, P. Phys. Rev. Lett. 104, 106408 (2010). 